\title{
Proposition de classification des syndromes malformatifs cranio-maxillo-faciaux
}

\author{
Antoine Delforge*, Gwenaël Raoul, Axel Wiss, Jean-Baptiste Kerbrat, Joël Ferri \\ Service de stomatologie et chirurgie maxillo-faciale, Hôpital Roger-Salengro, rue Emile-Laine, 59037 Lille Cedex, France
}

(Reçu le 16 mai 2010, accepté le 29 juin 2010)

MOTS CLÉS :

Syndrome malformatif cranio-facial / Classification physiopathologique

\section{KEYWORDS:}

Craniofacial syndrome /

Physiopathologic

classification

\begin{abstract}
RÉSUMÉ - La conduite orthodontique à tenir face à un patient porteur d'une malformation cranio-maxillo-faciale n'est pas formellement établie et ne fait à ce jour l'objet d'aucun consensus. Les auteurs proposent, au vu de l'ensemble des cas qu'ils suivent dans leur service et de la bibliographie actuelle sur chaque pathologie, de classer ces syndromes en quatre catégories physiopathologiques afin de conseiller pour chaque classe une prise en charge adaptée. En connaissant l'étiopathogénie d'une affection, on l'appréhende et la traite différemment. Plus de cinquante cas ont été répertoriés, mais il a été décidé de ne présenter que les pathologies les plus typiques sur le plan physiopathologique. Cette classification comprend les anomalies organiques d'une ou de plusieurs matrices fonctionnelles, les anomalies localisées des structures anatomiques, les anomalies généralisées du tissu mésenchymateux, les syndromes mixtes.
\end{abstract}

\begin{abstract}
A classification of cranio-facial syndroms. How to manage craniofacial malformative cases? It seems to be very difficult, especially in orthodontics because of the lack of consensus. The authors' aim is to propose a physiopathologic classification of these craniofacial syndromes in order to simplify the medical practice when we meet these patients. More than fifty cases are actually treated and followed in our hospital; we have described all of these cases before to choose the most representative in each category. These syndromes are classified in four categories, organ abnormalities of one or many functional matrix, localized abnormalities of the anatomical structures, general abnormalities of the connective tissue, mixed syndromes.
\end{abstract}

\section{Introduction}

L'extrémité céphalique concentre une diversité tissulaire considérable expliquant la variété infinie des tumeurs qui peuvent s'y développer. En termes de croissance, les interactions mécaniques, l'influence fonctionnelle, les facteurs génétiques y jouent tous des rôles importants variables dans le temps et dans l'espace. La richesse des influences explique la complexité des situations rencontrées et rend difficile la compréhension physiopathologique de certaines anomalies du développement craniocéphalo-rachidien [12]

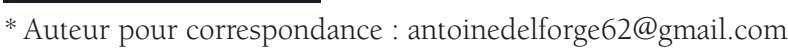

Il y a quelques années le Professeur Delaire avait souligné l'importance de la compréhension des grands syndromes cranio-faciaux quant à leur prise en charge en orthopédie dento-faciale, les analysant sous un oeil nouveau en rapportant ce qu'il appellera « les expériences naturelles de la pathologie » [3].

Nous souhaitons aujourd'hui proposer une classification des grandes anomalies du développement $\mathrm{du}$ pôle céphalique dans un but pratique, essentiellement adressée à l'orthodontiste afin que celuici puisse tout à la fois mieux reconnaitre, mieux pronostiquer et donc mieux traiter certains de ces cas dont la prise en charge est parfois si spécifique. Comprendre la pathologie pour mieux l'appréhender, telle est notre volonté. 
Mais classer c'est aussi simplifier parfois à l'extrême et donc se tromper. Ces erreurs sont probablement le prix à payer pour fournir un travail pratique utile au praticien qui pourra retrouver simplement les thérapeutiques adaptées à telle ou telle pathologie. C'est l'autre aspect de ce travail que de faire le point sur la prise en charge des syndromes répertoriés et d'en rapporter les consensus thérapeutiques.

Notre approche comprendra :

- les atteintes organiques et les dysfonctions de la matrice fonctionnelle,

- les atteintes organiques localisées,

- les atteintes organiques du tissu mésenchymateux et les maladies générales avec atteinte faciale, - les atteintes mixtes.

\section{Classification}

\subsection{Anomalies organiques d'une ou plusieurs matrices fonctionnelles}

Cette catégorie se caractérise par une atteinte lésionnelle organique (tumeur, trouble trophique, etc.) d'un effecteur majeur de la croissance cranio-faciale (matrice). La stimulation n'est donc plus adaptée, ce qui entraîne la dysmorphose.

Pratiquement toutes les matrices peuvent être affectées à un moment ou à un autre de leur évolution embryologique. Les raisons de ces atteintes sont nombreuses et variables, on peut citer sans être exhaustif les lésions d'origine vasculaire, traumatique, infectieuse ou toxique à l'origine de destruction ou d'insuffisance de développement tissulaire. Elles comprennent des accidents de parcours «simples» de l'embryogénèse comme par exemple la microphtalmie, jusqu'à des atteintes plus graves ne permettant pas le plus souvent de mener à terme la grossesse ou d'être à l'origine de mort néonatale, c'est le cas par exemple de l'anencéphalie qui est létale. Entre ces deux extrêmes, il existe une quantité considérable d'atteintes possibles comme par exemple les macro- ou microglossies, les anophtalmies, les torticolis congénitaux, les agénésies dentaires « simples », les imperforations choanales, les myopathies, etc.

\subsubsection{La scoliose}

\subsubsection{Description clinique}

La scoliose (scolios : tortueux en grec) est une déformation tridimensionnelle de tout ou partie de la colonne vertébrale (cervicale, thoracique ou lombaire) entraînant une torsion d'une ou de plusieurs vertèbres sur elle(s)-même(s) et provoquant une déformation du thorax, de l'abdomen et des zones para-vertébrales.

La différence entre scoliose et attitude scoliotique est importante puisqu'une attitude scoliotique correspond essentiellement à une inflexion latérale du rachis dans le plan frontal sans véritable torsion vertébrale et sans déformation asymétrique du tronc ou des zones para-vertébrales dans les trois plans de l'espace.

La scoliose la plus fréquente est de type idiopathique (70\% des cas) dont seulement 10 à $15 \%$ sont susceptibles d'évoluer vers une aggravation. Les scolioses idiopathiques touchent essentiellement les filles (5 à 6 filles pour 1 garçon). Il existe une prédisposition familiale (dans un cas sur trois, on retrouve un autre cas dans la famille).

En dehors des scolioses idiopathiques, il existe des scolioses secondaires, c'est-à-dire dues à des pathologies pouvant entraîner des déviations scoliotiques, comme les affections neuro-musculaires (myopathies, amyotrophies spinales...) ou neurologiques centrales (IMC, encéphalites, maladie de Recklinghausen, spina bifida, etc.). On parle aussi de scoliose « hypotonique » (liée à des paralysies ou des faiblesses musculaires).

\subsubsection{Atteinte maxillo-faciale}

Les anomalies sont de deux ordres :

- Secondaire à la scoliose avec une anomalie posturale retentissant sur le port céphalique à l'origine d'une dysmorphose asymétrique cranio-faciale, les déformations ne sont pas secondaires à la ventilation mais au port céphalique.

On note en effet fréquemment chez les enfants scoliotiques une classe III squelettique avec un articulé croisé latéral du côté de la scoliose et une déviation du point inter-incisif [13] (Fig. la à lc).

- Secondaire au traitement orthopédique (corset de Milwaukee ou tige de Harrington) à l'origine de classe III par propulsion mandibulaire : les enfants sont en appui mandibulaire constant sur ce corset et favorisent ainsi un excès de développement mandibulaire (en partie dû à l'hypotonie linguale associée) (Fig. 2a à 2c). 


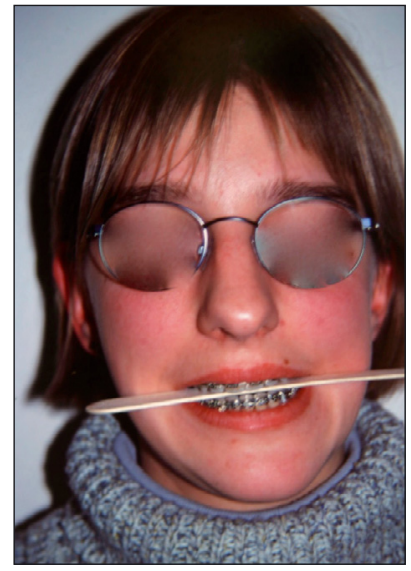

a

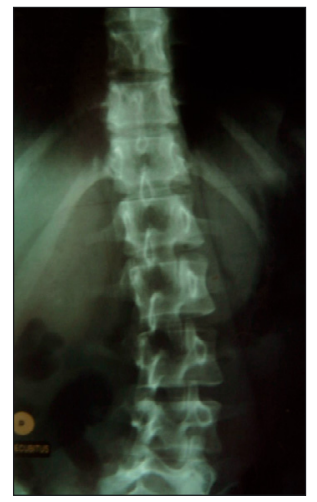

b

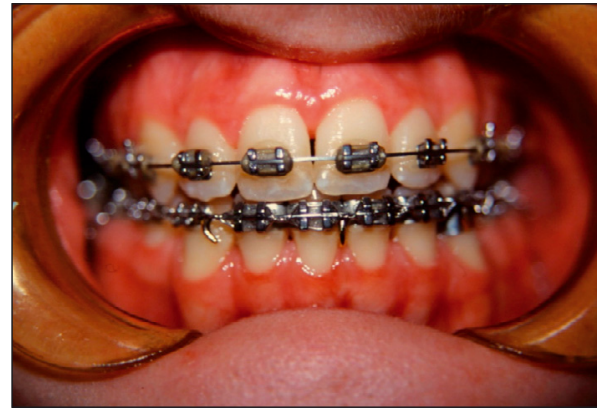

C

Figure 1

( $a, b$ et $c)$ Scoliose gauche : noter la tendance en classe III avec une latéromandibulie gauche et une bascule du plan d'occlusion.

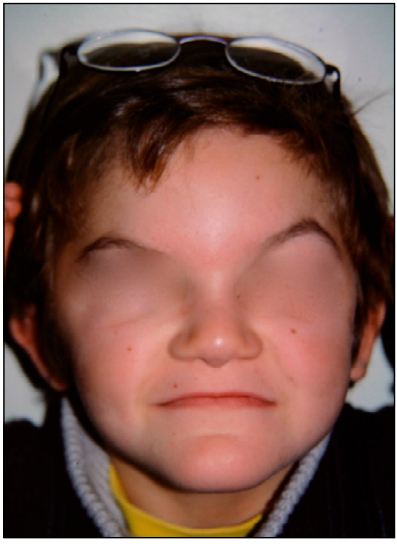

a

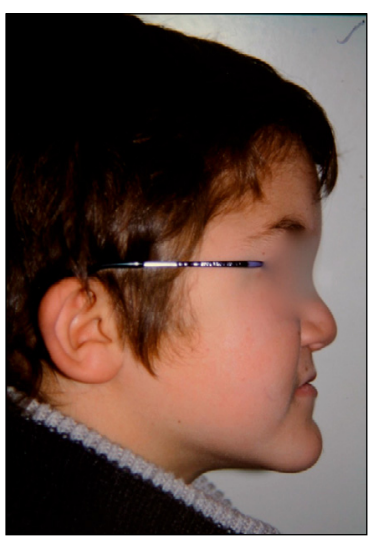

b

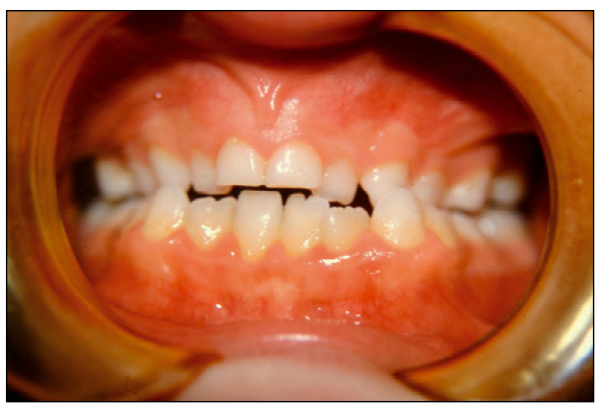

C

Figure 2

( $a, b$ et c) Enfant porteur d'un corset de Milwaukee avec une classe III dento-squelettique.

- En ce qui concerne la tige de Harrington, c'est le trouble de posture engendré par cette tige qui est responsable de malocclusion (Fig. 3a à 3c).

\subsubsection{Intérêt en $O D F$}

Le traitement est avant tout préventif par éviction de toute attitude vicieuse rachidienne et du port de tête [1]. Lorthopédie est intéressante avec le masque de Delaire (uni ou bilatéral). L'orthodontiste veillera à effectuer le saut d'articulé croisé latéral par une plaque palatine adaptée (ressort ou vérin sur la dent postérieure causale) plus ou moins associée à des cales molaires ou un plan de surélévation. Si besoin, un appareil multi-attache maxillaire et mandibulaire peut être collé pour la bonne correspondance des points inter-incisifs. L'enfant doit faire l'objet d'une surveillance accrue. Une rééducation posturale est systématique [13].

\subsection{Anomalies localisées des structures anatomiques, du mésenchyme}

Cette catégorie se caractérise par des atteintes organiques de l'os facial ou des effecteurs, et parfois des tissus mous associés, sur un territoire donné. Il s'agit de lésions territoriales le plus souvent rattachées à l'atteinte d'un élément embryologique précis. L'ensemble des tissus, quelque soit leur nature, est affecté. L'atteinte, toutefois, peut être variable avec des différences en fonction de la nature tissulaire. Pour certains, ces lésions entrent dans le cadre d'atteintes territoriales des crêtes neurales (Couly Le Lièvre). Ces anomalies de croissance initialement localisées, peuvent néanmoins avoir un retentissement plus im- 


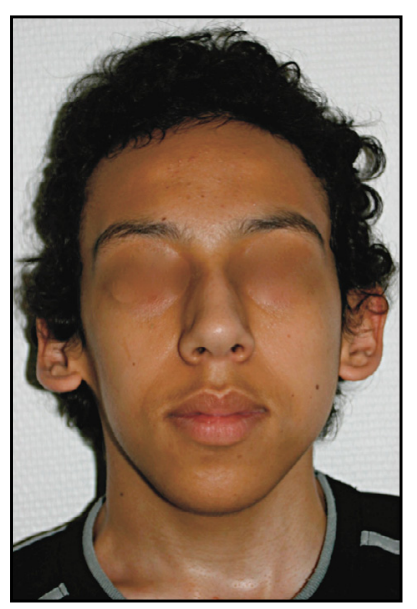

a

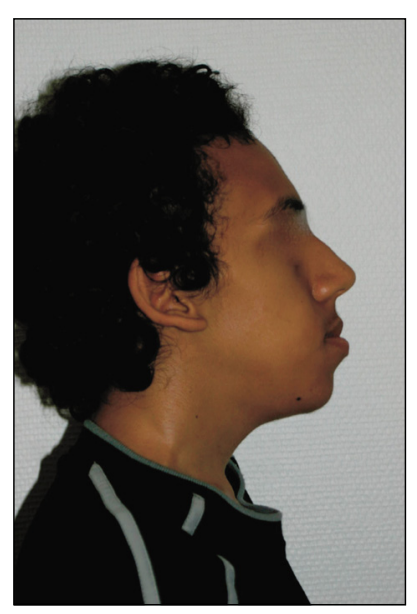

b

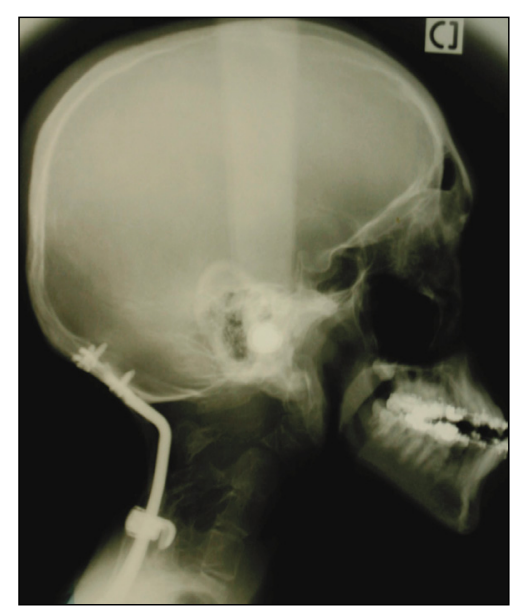

C

Figure 3

( $a$, b et c) Retentissement d'un « équivalent » de tige de Harrington : le patient est atteint d'une anomalie atloïdo-occipitale stabilisée en sous périosté par une tige mimant celle de Harrington. Le retentissement en classe III est patent.

portant. Ce retentissement étant d'autant moins important que l'on se trouve éloigné du centre organofonctionnel atteint [3].

\subsubsection{Les microsomies hémifaciales}

Ce terme a été consacré par Gorlin pour désigner un ensemble de malformations caractérisées par des insuffisances de croissance d'une hémirégion cranio-cervico-faciale. Dans ces affections, c'est l'ensemble des structures osseuses, musculaires, cutanées ou nerveuses qui peuvent être atteintes à des degrés divers. Les formes les plus simples se résument à une atteinte limitée de l'unité condylienne ou du pavillon de l'oreille qui apparaît réduit, les formes les plus graves affectent l'ensemble de l'hémistructure cervico-cranio-faciale avec paralysie faciale et surdité par absence d'appareil auditif (syndrome de Goldenhar). Pour les atteintes de l'unité condylienne qui intéressent en premier lieu l'orthodontiste, il faut rappeler que c'est Pruzansky qui a proposé une classification qui fait référence, trois types d'atteinte sont proposées [11] :

- Type I : raccourcissement unilatéral du ramus sans atteinte anatomique patente.

- Type IIA : le condyle et le ramus sont hypotrophiques, mais le condyle est normalement orienté par rapport à la cavité glénoïde qui est normale. Le processus coronoïde peut être absent.
- Type IIB : le condyle et le ramus sont raccourcis et déplacés mésialement. Il n'y a pas de cavité glénoïde fonctionnelle.

- Type III : absence complète du ramus, du condyle et du processus coronoïde.

Les microsomies hémifaciales comprennent le syndrome du premier arc et le syndrome de Goldenhar (forme plus grave), que nous décrirons successivement.

\subsubsection{Le syndrome du premier arc}

\subsection{Description clinique}

Le terme de microsomie hémi-faciale a été proposé en 1963 par Gorlin pour désigner l'association unilatérale d'une microtie, d'une microstomie et d'anomalies du développement du ramus et du condyle. La caractéristique fondamentale de ce syndrome est l'unilatéralité des anomalies faciales. La fréquence est d'environ 1 cas pour 3 à 6000 naissances. Le sex ratio est en faveur de l'homme [5].

Le faciès est d'emblée très évocateur : le menton est dévié du côté atteint.

Loreille externe peut manquer presque totalement et une atteinte des oreilles moyenne et interne est également possible. Langle mandibulaire est effacé. Les régions parotidienne, malaire et massétérine sont aplaties (Fig. 4a et 4b).

Latteinte mandibulaire siège sur la branche montante, et prédomine sur le condyle. Le corps mandibulaire se termine alors en arrière par une extrémité 


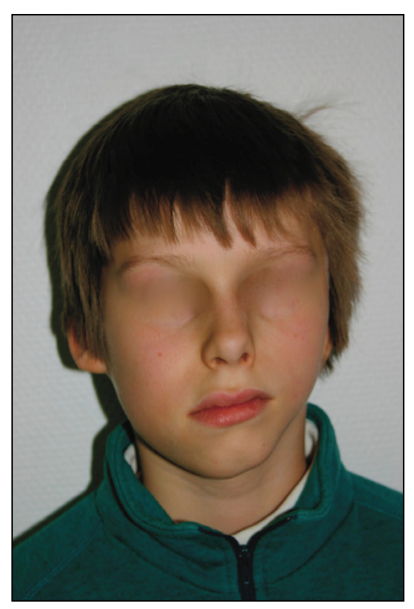

a

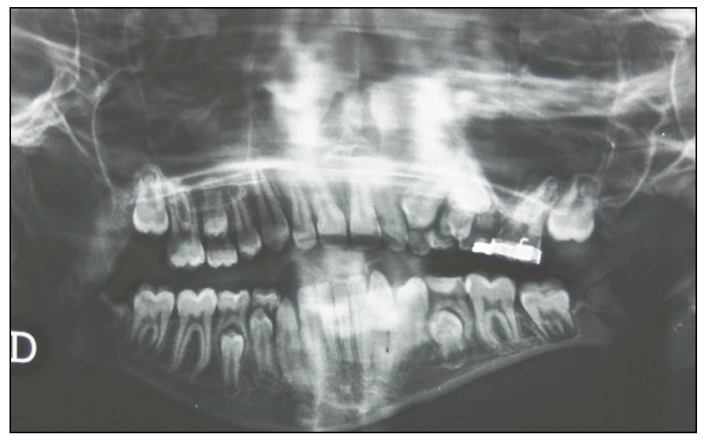

C

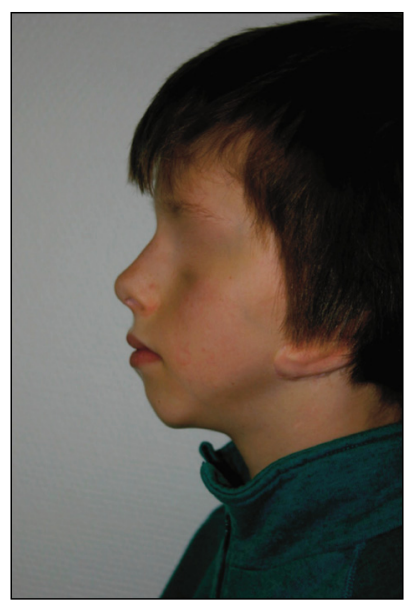

b

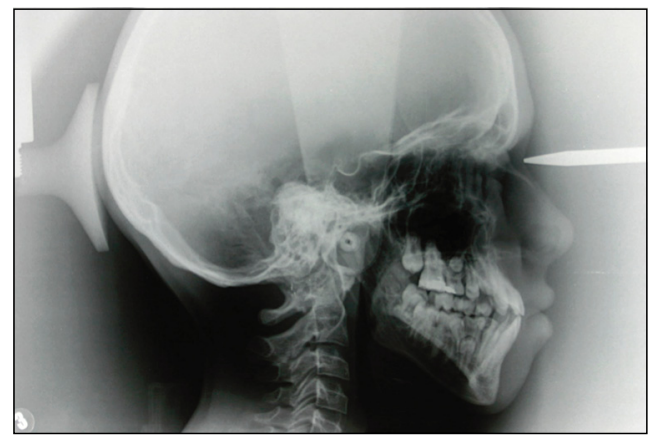

d

Figure 4

( $a, b, c$ et d) Syndrome du premier arc : noter la latéromandibulie et l'ascension de la partie postérieure du côté atteint.

en pointe correspondant à l'entrée du canal dentaire inférieur (Fig. 4c et 4d).

\subsection{Atteinte maxillo-faciale}

Les arcades alvéolaires présentent des anomalies de forme, de dimension et d'orientation avec dans les formes unilatérales: ascension de la partie postérieure du plan des molaires du côté affecté, obliquité transversale du plan d'occlusion, étroitesse de l'hémivoûte palatine et diminution de volume de la moitié correspondante de la langue. Dans $7 \%$ des cas, il y a une fente vélo-palatine. On ne peut parler de béance vraie dans ce syndrome, car la dysmorphose s'installe lentement laissant les compensations alvéolaires possibles.

\subsection{Intérêt pratique en ODF}

La prise en charge de ces patients est excessivement variable en fonction de l'âge de découverte, de l'atteinte et des équipes chirurgicales.
Lorthopédie est efficace dans le sens transversal, on utilisera les plaques d'expansion, les quadhélix ou les disjoncteurs. Certaines équipes utilisent un activateur de classe II unilatéral [2].

Lorthodontie assure le nivellement dentaire classique.

Pour nous, les choses peuvent se résumer de la façon suivante :

- Forme majeure du jeune enfant : nous préconisons une reconstruction par greffon chondrocostal. Celui-ci assure tout à la fois un traitement partiel de la dysmorphose et un centre primaire de croissance. Dans ces formes, et sous réserve d'une bonne prise du greffon costal, des séances de distraction peuvent être proposées. Elles ont toutefois beaucoup d'inconvénients: contrairement à ce qui a été espéré, elles n'augmentent pas la masse réelle des tissus mous (il est même probable qu'une fois la distraction arrêtée, les tissus aient tendance à un certain retour à leur situation 


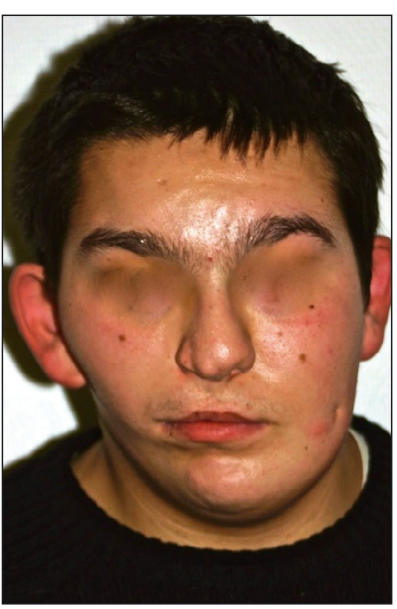

a

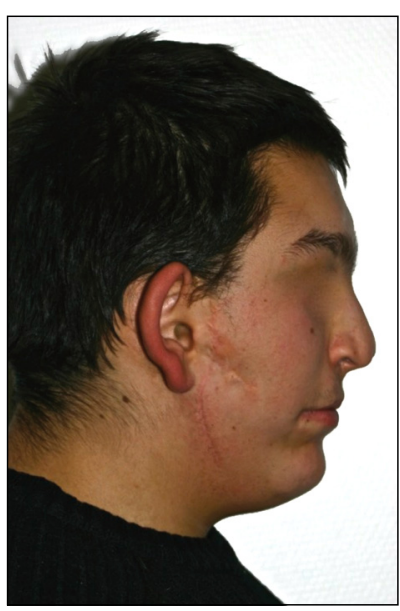

b

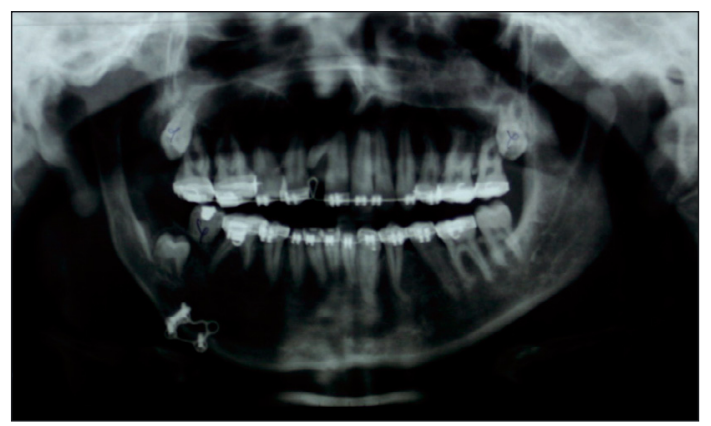

C

Figure 5

( $a$, b et c) Syndrome de Goldenhar : le patient a déjà bénéficié d'un greffon chondro-costal droit.

initiale) et les résultats occlusaux sont souvent aléatoires. De plus, chaque distraction nécessite deux interventions (pose et ablation du matériel), et il a été prouvé qu'en dehors de la distraction la croissance ne progresse pas $[7,9]$.

Tous ces éléments font que pour nous la distraction reste aujourd'hui exceptionnelle dans ces syndromes.

- Forme mineure de l'adolescent : chirurgie orthognathique réglée (ostéotomie unilatérale de type Epker).

On veillera toujours à préserver l'ATM atteinte, par exemple par une gouttière de surélévation occlusale.

\subsubsection{Le syndrome de Goldenhar}

\subsection{Description clinique}

Appartenant également aux microsomies hémifaciales, le syndrome de Goldenhar en est la forme la plus grave. Cette dysplasie oculo-auriculo-vertébrale (OAV) est caractérisée par une hypoplasie faciale asymétrique associée à des anomalies oculaires (dermoïde ou lipodermoïde épibulbaire, microphtalmie, colobome de la paupière supérieure...), une microtie, des appendices ou sinus pré-auriculaires et des malformations vertébrales (fusion des cervicales, platybasie, puzzle vertébral...). Latteinte faciale est généralement unilatérale, mais peut être bilatérale avec une expression plus sévère d'un côté $[4,6]$.

\subsection{Atteinte maxillo-faciale}

Latteinte est identique à celle décrite pour le syndrome du premier arc, mais plus grave, avec les deux étages de la face qui sont pathologiques et parfois une extension rachidienne (Fig. 5a à 5c).

\subsection{Intérêt pratique en ODF}

La prise en charge est la même que celle du premier arc avec néanmoins deux particularités :

- l'atteinte étant plus importante, les traitements sont souvent plus lourds,

- si un retard mental est associé, la réalisation des radiographies et l'adhésion à certains traitements sont parfois difficiles.

\subsection{Anomalies généralisées du mésenchyme}

Nous classons dans cette catégorie l'ensemble des anomalies du développement secondaire à un trouble constitutionnel du tissu conjonctif. Le tissu osseux pouvant être considéré comme du tissu conjonctif spécialisé, il est directement atteint. Il l'est également secondairement par atteinte des muscles qui sont eux aussi du tissu conjonctif spécialisé et qui, dysfonctionnant, auront un impact sur le développement osseux sous-jacent. Il y a une anomalie de la structure même du tissu osseux facial (il ne s'agit plus là d'une anomalie localisée mais bien d'un trouble généralisé constitutionnel).

C'est le cas pour de nombreuses pathologies telles que les dysostoses, les craniosténoses, les neurocristopathies... 


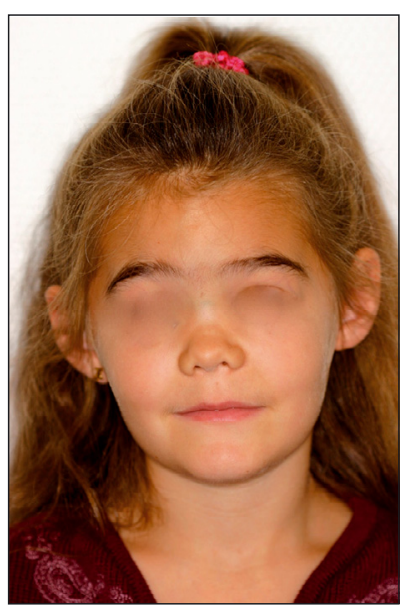

a

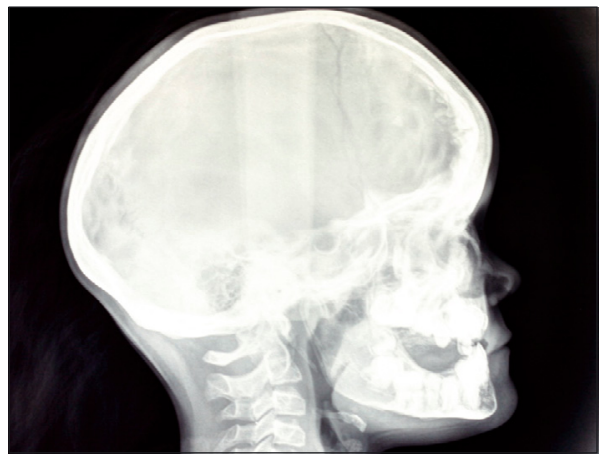

C

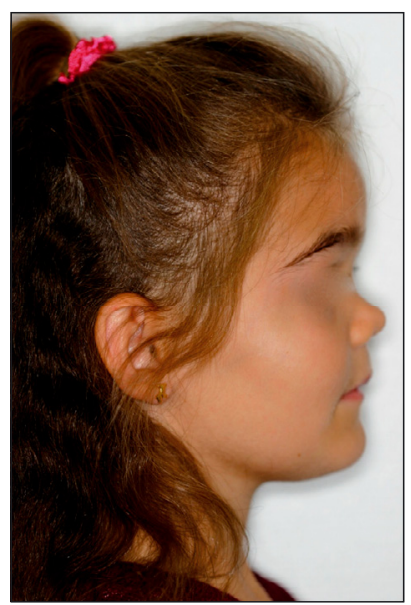

b

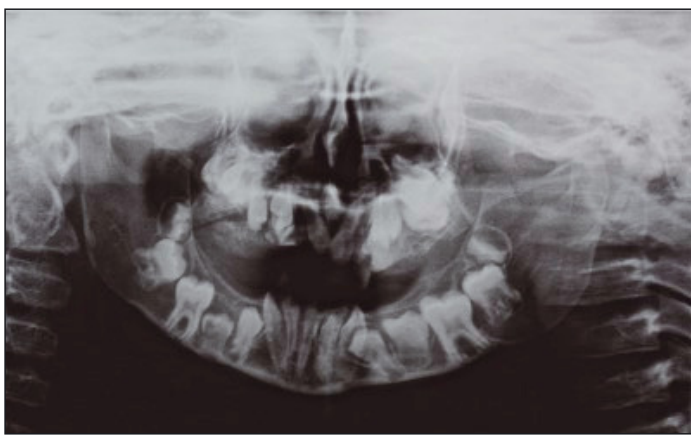

$\mathrm{d}$

Figure 6

( $a, b, c$ et d) Dysplasie fronto-métaphysaire : noter l'hypodontie et les inclusions dentaires.

Le nombre de maladies entrant dans cette catégorie est très important, il est vraisemblable qu'elles n'aient pas toutes encore été identifiées.

\subsubsection{Le syndrome de Pyle ou dysplasie fronto-metaphysaire}

\subsubsection{Description clinique}

La maladie de Pyle est une dysplasie osseuse caractérisée par un genu valgum, des anomalies métaphysaires avec élargissement des os longs touchant les diaphyses, et donnant au fémur et au tibia une apparence de flacon Erlenmeyer, un élargissement des côtes et des clavicules, une plastyspondylie et un amincissement cortical avec atteinte frontale. À ce jour, moins de 30 cas ont été rapportés dans la littérature. Le mode de transmission est autosomique récessif [10] (Fig. 6a et 6b).

\subsubsection{Atteinte maxillo-faciale}

Il y a peu de manifestations maxillo-faciales. On note un épaississement des os du crâne, principalement dans la région sus-orbitaire. Latteinte la plus fréquente est un retard d'éruption dentaire voire une hypodontie pouvant être grave (Fig. 6d). On observe parfois une promandibulie avec classe III d'Angle (Fig. 6c).

\subsubsection{Intérêt pratique en $O D F$}

Lorthopédie est peu efficace compte tenu du trouble osseux squelettique. Cependant, un masque de Delaire peut être proposé avant le pic de croissance, dans l'enfance [10]. S'il n'y a pas de décalage sagittal majeur, la compensation alvéolo-dentaire par orthodontie est efficace; en effet, l'os alvéolaire est sain. Cependant, la formule dentaire est variable d'un cas à l'autre. Si la classe III est importante, une chirurgie peut être envisagée à l'âge adulte. 


\subsection{Les syndromes mixtes}

Nous avons mis dans cette catégorie les syndromes caractérisés par des intrications complexes entre les anomalies malformatives et déformatives, et résultant de plusieurs mécanismes.

Ils peuvent ainsi combiner des anomalies localisées avec des troubles constitutionnels du tissu osseux ou encore des atteintes organiques d'une ou plusieurs matrices fonctionnelles. Ces combinaisons rendent difficiles la compréhension de leur physiopathologie. Néanmoins, l'individualisation de certaines particularités est possible et peut être rattachée à un caractère déformatif.

\subsubsection{L’acromégalie}

\subsubsection{Description clinique}

L'acromégalie est une maladie acquise liée à une sécrétion excessive d'hormone de croissance (somathormone, STH). Elle est caractérisée par une apparition progressive de modifications de l'aspect physique ou syndrome dysmorphique (concernant en particulier le visage et les extrémités) et des manifestations systémiques. La prévalence est estimée entre $1 / 140000$ et $1 / 250000$.

Le plus souvent, l'acromégalie est diagnostiquée chez l'adulte (âge moyen : 40 ans) et touche autant les hommes que les femmes. En raison de l'apparition progressive et insidieuse des symptômes, le délai entre le début de la maladie et le diagnostic est souvent de 4 à plus de 10 ans. Les principales caractéristiques cliniques de l'acromégalie sont des extrémités élargies (mains et pieds), des doigts agrandis, épaissis et boudinés et un épaississement des tissus mous. L'aspect du visage est caractéristique : un nez épais et large, des pommettes saillantes, un front bombé, des lèvres épaisses et des traits grossiers.

Los et la peau du front sont épaissis ce qui conduit parfois à des bosses frontales. L'acromégalie a également des conséquences rhumatologiques, cardiovasculaires, respiratoires et métaboliques.

\subsubsection{Atteinte maxillo-faciale}

Cette pathologie illustre bien la combinaison de plusieurs phénomènes:

- une réactivation de la croissance condylienne donnant un tableau d'hypercondylie bilatérale,
- une augmentation de la croissance périostée avec corticale épaisse et massive (Fig. 7d et 7e),

- une croissance de l'ensemble de la cavité buccale liée à la splanchnomégalie (macroglossie), qui explique l'augmentation de l'arche mandibulaire dans son ensemble (Fig. 7c).

Tout cela est à l'origine d'une classe III dentosquelettique par promandibulie avec rotation antérieure de Bjork [15]. Le faciès est caractéristique: face haute et élargie, massive, "bestiale», aspect triste et vieilli, hypertrophie de tous les éléments osseux, cartilagineux, et cutanés, avec nez proéminent et volumineux, oreilles énormes, arcades sourcilières et sourcils épais, pommettes massives (Fig. 7 a et $7 \mathrm{~b}$ ).

La macroglossie est considérable, gênant l'élocution, la mastication, la phonation (Fig. 7c).

À l'examen endobuccal, on note une hypertrophie alvéolaire avec diastèmes interdentaires, proalvéolie et édentation fréquente.

Les radiographies montrent un allongement $\mathrm{du}$ prémaxillaire, une promandibulie considérable avec ouverture de l'angle, allongement important et épaississement du col du condyle, un abaissement de l'échancrure sigmoïde, du sommet du coroné, de l'orifice d'entrée du canal inférieur, un épaississement des corticales et une augmentation du volume du menton osseux. Il y a une pro-alvéolie supérieure et inférieure avec vestibuloversion incisivocanine [14] (Fig. 7d et 7e).

Létude céphalométrique de cette pathologie est intéressante puisqu'elle montre bien le rôle et l'importance des phénomènes morphogénétiques secondaires, d'origine essentiellement périostée, dans le développement du crâne et de la face [3]

\subsubsection{Intérêt pratique en $\mathrm{ODF}$}

Lorthopédie est peu utilisée dans ce cas car les déformations apparaissent tard.

Lorthodontie est parfois indiquée pour les encombrements dentaires, et, si besoin, en préopératoire. Les tractions intermaxillaires sont inefficaces. Le traitement médical a permis de freiner l'évolution de la pathologie, mais les déformations restent cependant fixes et à surveiller. On veillera notamment à la bonne situation des ATM. Un panoramique de contrôle annuel est systématique. Une rééducation linguale, voire une glossectomie adjuvante de réduction sont parfois bénéfiques. Une fois 


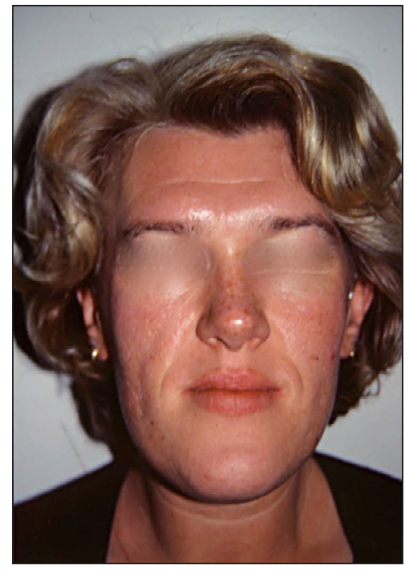

a

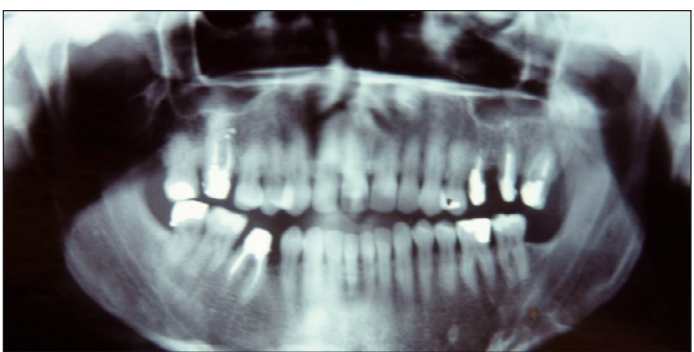

d

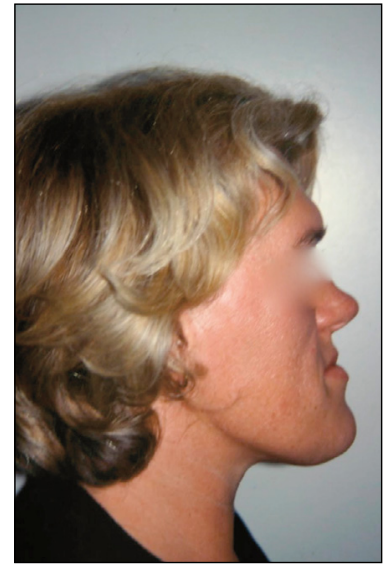

b

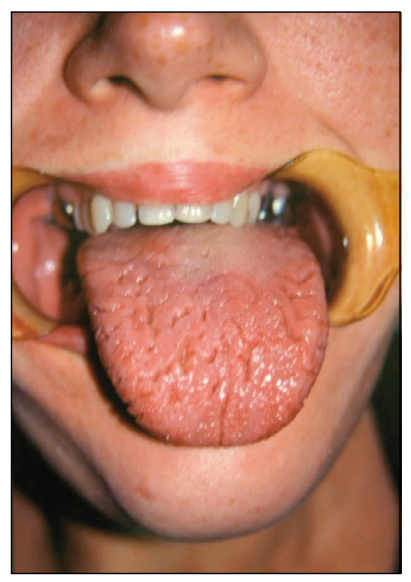

C

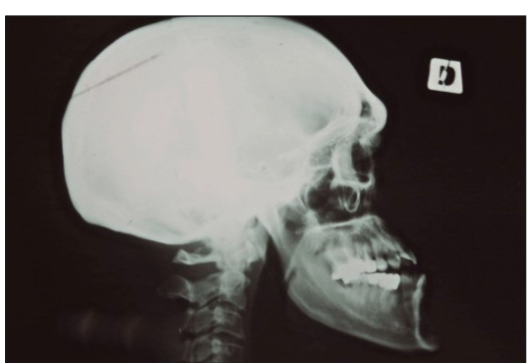

e

Figure 7

(a, b, c, d et e) Acromégalie : noter la macroglossie, la malocclusion de classe III, l'épaisseur de la corticale.

stabilisée, la dysmorphose en classe III peut faire l'objet d'une chirurgie orthognatique bimaxillaire qui s'avère souvent périlleuse compte tenu de l'épaisseur corticale $[8,15]$. La prise en charge est multidisciplinaire, et ces patients sont fréquemment suivis par les endocrinologues.

\section{Conclusion}

Ces différents syndromes suivis dans notre service ne sont qu'un échantillon de l'ensemble des malformations cranio-faciales qui existent. Certaines sont mieux connues que d'autres de par leur prévalence et leurs particularités. Cependant, cela montre la possibilité de créer des groupes physiopathologiques et ainsi de proposer une thérapeutique adaptée.

Trois écueils n'ont pu être évités : le manque d'exhaustivité, la difficulté de classer certains syndromes et l'absence de bibliographie pour les malformations rares ou peu connues.

Le but de ce travail est, pour le praticien, d'accéder à une conduite à tenir spécifique lorsqu'il est confronté à ce type de pathologie ou à une atteinte se rapprochant physiopathologiquement de celles décrites.

Pour conclure, il serait intéressant de compléter de manière prospective ce catalogue afin de couvrir un maximum de pathologies et ainsi de faciliter encore l'approche orthodontique de ces syndromes malformatifs.

\section{Remerciements}

$\mathrm{Au}$ Pr Delaire pour les informations tirées de son traité, et aux Drs Sebille et Baralle pour leurs conseils pratiques.

\section{Bibliographie}

[1] Ben-Bassat Y, Yitschaky M, Kaplan L, Brin I. Occlusal patterns in patients with idiopathic scoliosis. Am J Orthod Dentofacial Orthop 2006;130:629-633.

[2] Cascone P, Gennaro P, Spuntarelli G, Iannetti G. Mandibular distraction: evolution of treatment protocols in hemifacial microsomy. J Craniofac Surg 2005; 16:563-571.

[3] Ferri J, Bennani K, Sebille S, Caprioli F. Croissance réponses à la pathologie. Étude préliminaire. Orthod Fr 2000;71:241-248. 
[4] Fiore C, Santoni G, Lungarotti S, Signorini E. Goldenhar syndrome (a case report). Ophthalmologica 1983;186:162-168.

[5] Hartsfield JK. Review of the etiologic heterogeneity of the oculo-auriculo-vertebral spectrum (Hemifacial Microsomia). Orthod Craniofac Res 2007;10:121-128.

[6] Jena AK, Duggal R. Atypical Goldenhar syndrome: a case report. J Clin Pediatr Dent 2006;31:118-122.

[7] Lesne B, Bettega G, Morgon L, Aknin JJ. Distraction symphysaire et orthodontie. Orthod Fr 2008;79:197-207.

[8] Maeda A, Suzuki H, Irie M, Morita S, Sasaki M, Inokuchi $\mathrm{T}$, et al. [Surgical-orthodontic correction of mandibular prognathism due to acromegaly - a case report]. Nippon Kyosei Shika Gakkai Zasshi 1985;44:375-387 (en japonais).

[9] Molina F. Mandibular distraction osteogenesis: a clinical experience of the last 17 years. J Craniofac Surg 2009;20 Suppl 2:1794-1800
[10] Oppenheimer C, Oliveira BC, Sogabe M, Sanvito W. [Pyle's syndrome: report of a case.] Arq Neuropsiquiatr 1996;54:120-123 (en portugais).

[11] Pruzansky S. Anomalies of face and brain. Birth Defects Orig Artic Ser 1975;11:183-204.

[12] Radlanski RJ, Renz H. Genes, forces, and forms: mechanical aspects of prenatal craniofacial development. Dev Dyn 2006;235:1219-1229.

[13] Segatto E, Lippold C, Vegh A. Craniofacial features of children with spinal deformities. BMC Musculoskelet Disord 2008;9:169-179.

[14] Takakura M, Kuroda T. Morphologic analysis of dentofacial structure in patients with acromegaly. Int J Adult Orthodon Orthognath Surg 1998;13:277-288.

[15] Yagi T, Kawakami M, Takada K. Surgical orthodontic correction of acromegaly with mandibular prognathism. Angle Orthod 2004;74:125-131. 